\section{The Internet Book of Gene Therapy:}

Cancer Therapeutics

\section{edited by Robert E. Sobol and Kevin 1. Scanlon \\ Appleton \& Lange \\ ISBN 0-8385-3101-9}

\section{Reviewed by Paul D. Robbins \\ Department of Molecular Genetics and Biochemistry \\ University of Pittsburgh School of Medicine Pittsburgh, PA 15261}

The Internet Book of Gene Therapy: Cancer Therapeutics represents one of several publications focusing on the rapidly developing area of gene therapy applications and techniques, but was the first to use the electronic medium for publication. The book is published in an electronic version on the Internet - meant to be frequently updated - and a standard printed format. The content of the twenty-seven chapters varies, with some chapters being more review-oriented, whereas others more focused on discussing results of specific animal or clinical experiments. The book is highly informative with regards to both basic biology and applications of gene therapy to treating cancer.

The printed version of the book was published in 1995 and includes five sections. The first contains research and review articles on viral vector systems for gene therapy, including chapters on adenoviruses, adeno-associated virus, herpes simplex virus and cationic liposomes, as well as methods for safety testing of viral vectors for clinical applications. One inherent problem with publishing any collection of reviews on a diverse field like gene therapy is the ability to encompass all existing gene delivery systems, as well as their applications. The editors have done a commendable job of including reviews of the most widely used systems by leaders in the field of gene transfer, but have omitted, due to space constraints, less used vector systems such as lentiviruses, bioballistics, DNA conjugates and plus-strand RNA viruses. The first chapter by Douglas Jolly does provide an excellent overview of the strengths and weakness of each system, and of the current status in the development of different viral vectors.
The second section comprises the major part of the book and focuses on procedures for treating cancer using gene therapy. Its twenty chapters cover antisense and ribozyme approaches, drug resistance and drug sensitivity gene therapies, hematopoietic gene transfer including marking studies, immunotherapy, targeted therapy, tumor suppressor gene therapy and methods for the treatment of specific tumors. The different immunotherapy systems for treating cancer are reviewed by Sobol et al. However, it is surprising that the two chapters specifically dedicated to im-

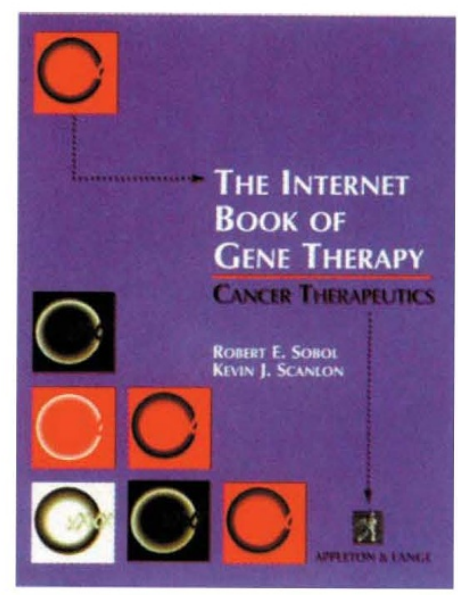

munotherapy approaches focus on IL-2and IL-7-mediated gene therapies since results with GM-CSF, interleukin12 and HLA-B7 have given more pronounced therapeutic effects in animal models and clinical trials. This is not a criticism, but rather another indication that it is difficult to include all relevant gene therapy approaches for treating a complex disease such as cancer in one book.

Different gene therapy trials from around the world and categorized by disease make up the third section. This is useful, allowing one to quickly identify which types of gene therapy trials are being conducted for cancer as well as other diseases. Given the number of recently approved trials, this section should be updated monthly. Unfortunately, the latest revision of the electronic version of the book dates back to June 1996.

The fourth section describes filed gene therapy patents published in 1995, also indicating the company and/or academic center who owns patent rights. Scientists working at biotechnology companies will find this section particularly useful. However, this section also requires updating and currently reflects only those patents that have been published due to filing in Europe or Japan.

Finally, U.S. regulatory guidelines for gene therapy, including "Points to Consider" for gene transfer protocols, somatic cells/gene therapy, cell lines, and products of bovine and ovine origin are considered in the last section. Also included is a discussion of the Food and Drug Administration (FDA) recommendations on Mycoplasma testing. These FDA and Recombinant DNA Advisory Committee (RAC) documents are readily available through other sources and thus do not add significantly to the book. It would be worthwhile for the editors to consider establishing links to FDA and $\mathrm{NIH}$ web sites to allow access to current information regarding gene therapy regulations.

Does the electronic version of the book work to facilitate easier access and continued updates? The answer is yes and no. The format of both hard and electronic versions is clearly the direction most review and methods books in science will be taking. In theory, the benefit of electronic publishing on the Internet is to allow continued updates and additions to the book prior to or in lieu of publication of subsequent printed editions. The Internet Book of Gene Therapy has not been updated recentlya major deficiency with regards to sections on patents and clinical trials. However, it appears as though the book will be updated soon, and possibly several links to related sites established. In addition, three new chapters are being added. Importantly, I encountered another problem-one which continues to plague the Internet: the server not responding.

In summary, The Internet Book of Gene Therapy: Cancer Therapeutics provides a good overview of the approaches for treating cancer using gene transfer. The electronic version, although it hasn't fully exploited the advantages of Internet publishing, represents the future of science books and journals. If the editors and publisher can indeed periodically update the book, include both new and modified chapters and establish links to related sites, then the book will continue to provide the reader/browser with an up-to-date overview of cancer gene therapy. 